\title{
Agent based Personalized e-Catalog Service System
}

\author{
M.Thangaraj, \\ Department of Computer Science \\ Madurai Kamaraj University ,Madurai, \\ Tamilnadu
}

\author{
M Chamundeeswari, \\ Department of Computer science \\ V.V.V College for Women \\ (Affiliated to Madurai Kamaraj university) \\ Virudhunagar, Tamil Nadu
}

\begin{abstract}
With the emergence of the e-Catalog, there has been an increasingly wide application of commodities query in distributed environment in the field of e-commerce. But e-Catalog is often autonomous and heterogeneous, effectively integrating and querying them is a delicate and time-consuming task. Electronic catalog contains rich semantics associated with products, and serves as a challenging domain for ontology application. Ontology is concerned with the nature and relations of being. It can play a crucial role in e-commerce as a formalization of e-Catalog. User personalized catalog ontology aims at capturing the users' interests in a working domain, which forms the basis of providing personalized e-Catalog services. This paper describes a prototype of an ontology-based Information retrieval agent. User personalized catalog ontology aims at capturing the users' interests in a working domain, which forms the basis of providing personalized e-Catalog services. In this paper, we present an ontological model of e-Catalogs, and design an Agent based personalized e-Catalog service system (ABPECSS), which achieves match user personalized catalog ontology and domain e-Catalog ontology based on ontology integrated
\end{abstract}

Keywords: personalization, semantic web, information retrieval, ontology, re-ranking algorithms, knowledge base ,user profile,ecatalog

\section{INTRODUCTION}

As Internet technologies develop rapidly, companies are shifting their business activities to e-Business on the Internet. Worldwide competition among corporations accelerates the reorganization of corporate sections and partner groups, resulting in a break of the conventional steady business relationships. For instance, a marketplace would lower the barriers of industries and business categories, and then connect their enterprise systems. Electronic catalogs contain the data of parts and products information used in the heavy electric machinery industry. They contain not only the commercial specifications for parts (manufacturer name, price, etc.), but also the technical specifications (physical size, performance, quality, etc.). Clearly defined product information is a necessary foundation for collaborative business processes. Furthermore, semantically enriched product information may enhance the quality and effectiveness of business transactions. As a multifunctional applied system, it serves for advertisement, marketing, selling and client support, and at the same time it is a retail channel.

As the number of Internet users and the number of accessible Web pages grow, it is becoming more and more difficult for users to find documents among e-Catalogs that are relevant to their particular needs. Users can search with a search engine which allows users to enter keywords to retrieve e-Catalogs that contain these keywords. The navigation policy and search have their own problems. Indeed, approximately one half of all retrieved documents have been reported to be irrelevant. The main reasons for obtaining poor search results are that (1) many words have multiple meanings (2) key words are not enough to express the rich concepts and the natural semantics of customers' queries. (3) The property query lacks of semantic support, and is difficult to search for knowledge, and has other problems of mechanisms. (4) Related merchandises cannot be returned. What is needed is a solution that will personalize the e-Catalog selection and be presented to each user. A semantically rich user model and an efficient way of processing semantics are the keys to provide personalized eCatalog services. In view of the existing limitations, we develop a personalized ontology based on user model, called user personalized catalog ontology, which has the same level of semantics as domain ontology.

The rest of this paper is structured as follows: Section 2 describes related work. Section 3 , explains the theory of propose system. Section 4 we put forward our modeling methodology for generating user personalized catalog and product domain ontology. Then in Section 5, we present the implementation of the system and its evaluation. Conclusion and future work are drawn in Section 6

\section{RELATED WORK}

E-catalogues play a critical role in eprocurement marketplaces. They can be used in both the tendering (pre-award) and the purchasing (post-award) processes. Companies use e-catalogues to exchange product information with business partner's .Suppliers use ecatalogues to describe goods or services that they offer for sale. Mean while buyers may use e-catalogues to specify the items that they want to buy $[1,2]$ Matching a product request from a buyer with products e-catalogs that have been provided by the suppliers, helps companies to reduce the efforts needed to find partners in e-marketplaces $[5,7]$

\subsection{E-Catalog Ontology Design}

Researches in recent years show that applying ontology to e-commerce scenarios would bring benefits such as solving the interoperability problems between different e-commerce systems [3, 4]. Especially, eCatalog, which is a key component of e-commerce systems, 
seems to be the most adequate domain within e-commerce scenarios where ontology can realize the expression of eCatalog on a semantic level. It is possible for e-business systems to offer diverse interoperable services by sharing well-defined e-Catalog model containing rich semantics. Fensel [5] described in principle how ontology's can support the integration of heterogeneous and distributed information in ecommerce scenarios which is mainly based on product catalogs, and what tasks are needed to be mastered. ECatalog ontology model is defined as ECO (concepts, relationship, properties, axioms and individuals).

The traditional key-based retrieval method cannot satisfy massive heterogeneous personalized catalog service, then [8] introduce meta search engines, but this method is passive service. [9] Provided an intelligent catalog recommend method using customer requirements mapping with product categories. [10] Brought forward personalized eCatalog model based on customer interests and [11] is a personalized catalog service community, WebCatalog [12] designed enterprise e-Catalog based on customer behavior. The knowledge representation and acquisition of client catalog turns into the key problems. In order to reach an effective method, K-clustering algorithm and e-Catalog segmentation approach are described in [13] and [14] described the customer segmentation method based on brand and product, price. In [15] the author researched personalized catalog service with one-to-one market by association rules and CART. In recent years, personalized ontology's (also known as private ontology, such as [9] are introduced into eCatalog service, Peter Haase put forward personalized ontology learning theory based on user access and interest coordination [16]. In distributed system, there are sharing concepts of domain ontology's and personalized knowledge ontology's [17]. Therefore, it has important theoretical and practical significance to apply personalized ontology's to personalized e-Catalog service.

\section{PROPOSED ARCHITECTURE}

The personalized information retrieval system based on multi-agent adopts the working fashion of multi-agent cooperation, multi-agent collaborate mutually and communicate to one another for accomplishing task.

The system consists of User Agent, Query Generation Agent, Reasoning and Expanding Agent, Searching Agent and Filtering Agent, Personalized Ranking Agent and Knowledge Base. It is shown in Figure 1.[23] All agents are monitored entirely to fulfill proprietary system functions, including information retrieval and Knowledge Base undate

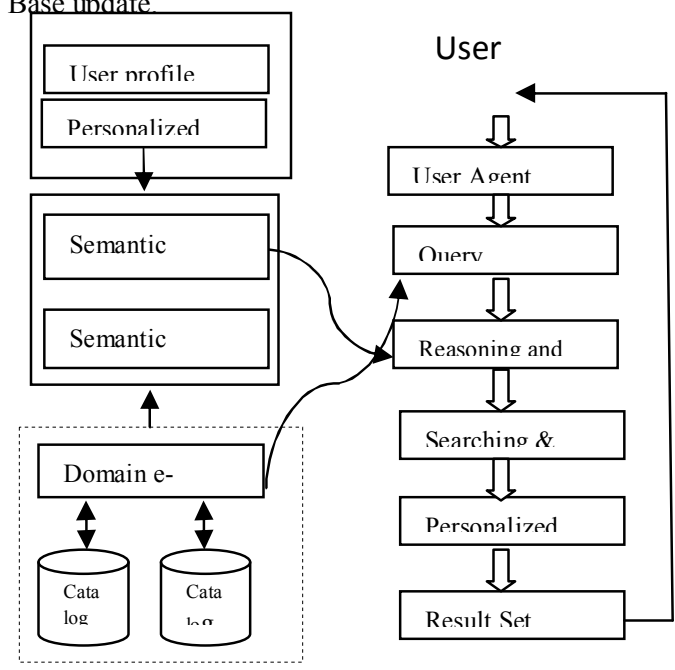

Figure 1 Architecture of Agent based personalized e-Catalog service system (ABPESS)

(1) User Agent: User Agent is the mutual interface between user and system, and provides a friendly platform to users. User Agent also takes over result from Personalized ranking agent and presents personally these results to user. User's browsing or evaluating behavior can be stored and learned by User Agent, so user interest model may be updated and improved in time.

(2). Query Generating Agent: QGA incepts user's retrieval request, which is transformed to prescriptive format, and transmits the formatted user request to Reasoning and expanding agent.

(3) Reasoning and expanding agent: In the personalized information retrieval system, Reasoning and expanding agent takes charge of receiving formatted user request from QGA, and the user request is expanded according to user interest model. Afterwards, the perfected user request is transmitted to Searching \& Filtering Agent.

(4) Searching Agent and Filtering Agent: Searching Agent collects all data from initiative Searching Agent or metaSearching Agent, takes out invalid links, deleting excrescent information, and finally processed data are transmitted to Personalized Re-ranking agent . Filtering Agent analyses the returned data from Searching Agent, filtrating useless information, and processed results are send to Personalized Re-ranking agent.It also completes search result statistic, user browse statistic, and retrieval keywords statistic, etc. Various statistic outcomes are stored in Knowledge Base.

Algorithm of e-Catalog- searching and filtering:

Constructing semantic results SR, where DO is domain ontology, expanding ontologies, SRD is $\mathrm{r}$.

Keyset $\mathrm{KS}=\left\{\mathrm{k}_{1}, \mathrm{k}_{2} . . \mathrm{k}_{\mathrm{n}}\right\}$

Input: keyword, basic ontology DO;

Output: semantic results SR;

Search(KS,DO)

\section{Begin}

for(each KS) \{finding DO mapping $\mathrm{K}_{\mathrm{i}}$, according to the semantic mapping table; \}

for(sub-ontology s in DO)

if $\left(R^{w}{ }_{d}(\mathrm{Oi}, \mathrm{s}) \geq \mathrm{m} \& \& \mathrm{~s}\right.$ isn't in DO)

find the result s for semantic query

copy the components of $\mathrm{s}$ to SR;\}

return SR;

End

(4) Personalized Re-ranking agent : it is the decisionmaking center of personalized information retrieval system based on multi-agent, and assorts with data communication and task assignment. Personalized Re-ranking agent use reranking alg. To find the new score based on user interest.

$P R$ (uid)

Begin

If uid exits $\{\quad$ Re-ranking(CP, uid,interest $)\}$

else

\{ 


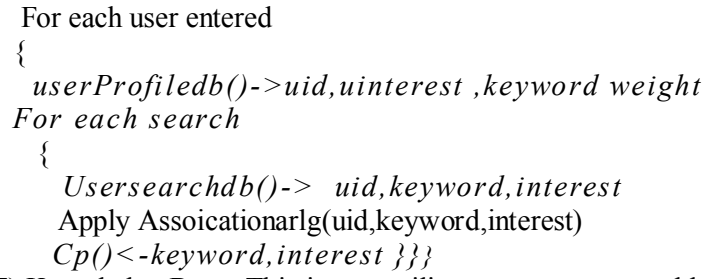
representation, and even provides machine translation tools resolving cross-lingual dependencies.

(6) Semantic ontology: It contains some product knowledge used to generate the queries. It was designed as a hierarchical tree, with a frame based representation approach. This ontology must be at some degree context free, but it has to point elements of the search engines used by the Query Generation module.

\section{METHODOLOGY:}

\subsection{Method of Designing User Catalog ontology:}

In order to satisfy customer's personalized requirement, we should master more information of the customers. Sometimes customers also cannot describe their own thought, to understand their potential mind, we need user e-Catalog ontology. Based on consumer behavior, we propose a personalized approach to build personalized catalog ontology (PCO).

PCO supposed to be formed by

$>$ First, build user personal ontology (PCO) based on users' personal information and preferences

$>$ Second, extract user catalog information from user purchase history, user searching keywords, user browsing catalog, user feedback information

$>$ Third, web resource according to user catalog ontology information

Agent based e-catalog organizes a group of keywords expressing users' interest through PCO, when users puts semantic query, it is no longer a simple keywords match, but considering users' personal preference and information, and tightly integrates the users and products, so that the system can improve the semantic query precision rate and recall rate, as well as be conducive to sort query results.
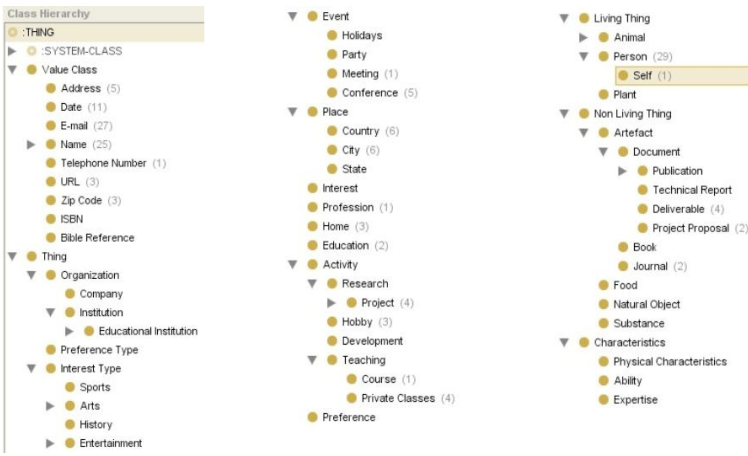

Figure 2 framework of user Personalized Catalog Ontology

Figure 2 shows a user catalog ontology framework, in which we describe user interest information, user preference and product concepts, properties and individuals that users are interested in, including product area, brand and quality authentication. Users associate with the product by property hasPreference, and we set aside a weight interface in property "has Preference", indicating the fact users' different observation extent about different properties of a product which is shown in Figure 3.

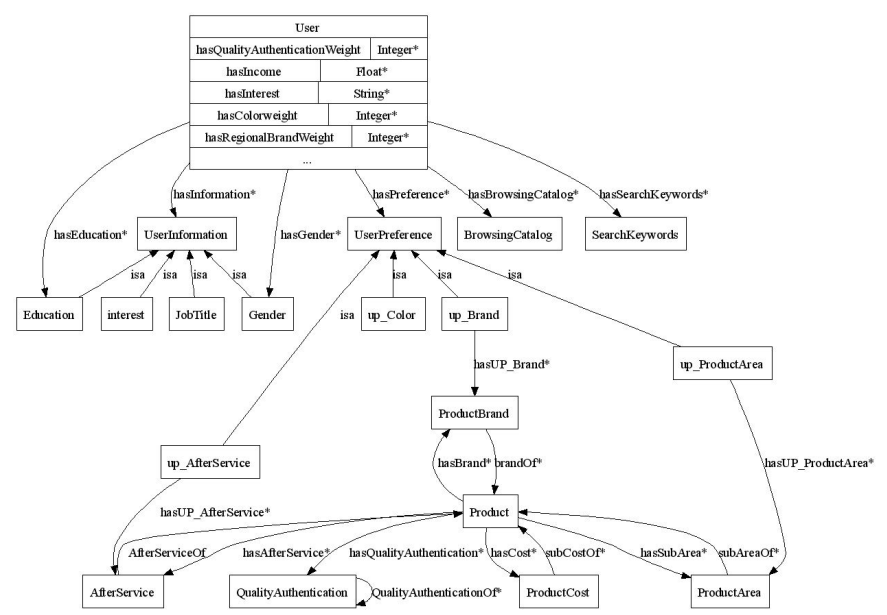

Figure 3 The Relationship of user Personalized Catalog Ontology

Generating Semantic Catalog ontology (SCO):

Generation domain e-Catalog ontology is divided into three steps:

$>$ Extraction of the core concepts and properties for domain e-Catalog ontology's, according to the UNSPSC standards, wordNet standards and semantic catalog dictionary.

$>$ Construction of a SCO model..

$>$ Acquisition standardized DECO by e-Catalog ontology pruning subsystem, combining WorldNet and semantic catalog dictionary. 


\subsection{Semantic Match Based on Ontology}

One critical step of semantic match is that calculation semantic match degree between the terms of ontology concepts. There have been many methods to calculate conceptual semantic match in e-commerce scenarios [18]. Common calculation methods and models are: (1) Identifierbased method [19], which uses word-building to find the semantic match degree between the concepts, and primarily reflects the linguistic similarity of the two concepts; (2) Synonym dictionary-based method [20], which organizes all concepts to a tree hierarchy structure according to synonym dictionary where there is only one path between any two nodes and this path length is taken as a measure of semantic distance of the two concepts; (3) Feature Match-based model [21], which calculates semantic match of concepts by the collection of properties; and (4) Semantic relationship-based model [22], also known as the semantic distance-based model, which calculates semantic match of concepts based on hierarchy information and is mainly used in the same ontology. In this paper, we need to calculate the semantic match of UPCO and DECO using Individual-based Semantic Match methods.

\subsection{Individual-based Semantic Match}

To query user preferences product, we should get the product similar with user preferences, namely calculating the instance similarity between SCO individual and PCO individual. We calculate the semantic match of the individuals by the property value-based method.

calculate the semantic match method based on linguistics, when we calculate semantic match degree of the property values

$$
\operatorname{smi}\left(C_{1}, C_{2}\right)=\frac{e d\left(C_{1}, C_{2}\right)}{\left(\left|C_{1}\right|+\left|C_{2}\right|\right) / 2}
$$

\section{Explanation:}

$\left|C_{1}\right|$ is the length of the string $C_{1,}\left|C_{2}\right|$ the length of the string $C_{2}, e d\left(A, C_{2}\right)$ is the same number of characters in $C_{1}$ and $C_{2}$.

String $C_{1}$ and $C_{2}$ are input parameters, in the process, which are the properties values of two products calculate the individual semantic match of the two products through comparing several groups property semantic match degree.

\subsection{Basic function of ABPECSS}

To implement agent based E-service first of all, personalized user catalog ontology's are customized according to consumers(PCO) ; secondly, we need to build domain e-Catalog ontology's(SCO) ; thirdly, we match the two kinds of ontology's by match algorithm through semantic reasoning and expanding agent which generates match result sets.

The basic the theory of distributed semantic query based on e-Catalog ontology is: users input key words, phrases, sentences or paragraphs (users' queries, $\mathrm{U}_{\mathrm{q}}$ ) in user querying interface; query generator module translates $\mathrm{U}_{\mathrm{q}}$ to ontology descript; query reasoning and expanding module is responsible for reasoning and expanding the descript using the semantic match result set is, then outputs semantic queries $\left(\mathrm{S}_{\mathrm{q}}\right)$ in forms of Sparql and finally extract data from distributed e-Catalog database. Searching and Filtering module combines the distributed results and filters repetitive and invalid results .personalized ranking agent rearrange the result sets and recommended to the user.

\subsection{Results Personalization}

The personalization helps in getting relevant results for the user's query. As shown in the queryprocessing steps, the personalization starts with the query enrichment step, where we utilize the user profile to expand the query and to fill in the incomplete query templates. Here, we go into more detail with the results personalization steps and show how we capture the user's feedback.

Results personalization steps

Personalizing the results involves presenting the results in the most effective way possible through several steps. The first step is answering the user's query in the same language he asks it in, regardless of the language of the ontology and the knowledge base, which has the annotated data. The second step is answering the user's query in appropriate syntax based on the question type; a confirmation question is different than a subjective question, as the user expects a "yes" or "no" answer in the first type, while $\mathrm{s} / \mathrm{he}$ expects a list of items in the second type. So, an answer is personalized to express the understanding of the query and to be familiar to the user. The third step is ranking the results based on the user's preferences and interests. Finally, it filters the non-relevant food or health information based on the user profile.

\subsection{User's feedback}

Continuous feedback collection is required to sharpen the user's experiences. Feedback is not only explicit, but also implicit, as it can be collected through different measures. Many measures could help in reflecting the implicit feedback, such as time spent in browsing the results, clicks on the data sources, clicks on the result facets related to the search results, etc. All interactions and feedback are recorded and logged in the usage log which is analyzed after each query to know how effective the results are and how we can improve the future recommendations. This is reflected in the user profile ontology

\section{IMPLEMENTATION AND EXPERIMENTATION}

In this section experiments carried out to evaluate the performance of proposed system will be discussed from a quantitative point of view by running some experiments to evaluate the precision of the results. The basic idea of the experiment is to compare the search result from keyword based search engine with proposed one on the same category and the same keywords.

The proposed system ABPECSS is implemented in C\#.Net as Web-based system using Visual Studio 2008, .NET Framework 3.5, and SQL Server 2005. The system was evaluated by having 20 users implement the system to create personal ontology's. The user was given a query interface to 
input his/her query parameters and view each one of their concepts and every concept from the SCO that had been matched to the personalize catalog concept. Also the user was able to decide which concept or property was not needed when reasoned and expanded the query. In the experiment, we take different electronic items as an example. The user was asked to compare the semantic query result and that from the keyword-based search engines and decide if ABPECSS was the better. Therefore, we manually create the domain eCatalog ontology (SCO) and user personalized catalog ontology (PCO) and calculate semantic match degree in the system.

Table 1 Experimental results statistics for query manipulation

\begin{tabular}{|l|l|l|l|l|l|}
\hline concepts & $\begin{array}{l}\text { Total } \\
\text { found } \\
\text { concept } \\
\text { s }\end{array}$ & $\begin{array}{l}\text { concept } \\
\text { Found } \\
\text { correct }\end{array}$ & $\begin{array}{l}\text { Correct } \\
\text { concepts } \\
\text { manually }\end{array}$ & Precision & Recall \\
\hline $\begin{array}{l}\text { Dell Inspiron 15R } \\
\text { i3531-1200BK }\end{array}$ & 89 & 71 & 74 & $91.36 \%$ & $\mathbf{8 0 . 4 3 \%}$ \\
\hline $\begin{array}{l}\text { Dell Alienware 18 } \\
\text { Gaming Laptop. }\end{array}$ & 89 & 71 & 93 & $71 \%$ & $5.54 \%$ \\
\hline $\begin{array}{l}\text { Canon EOS 6D Black } \\
\text { SLR Digital. }\end{array}$ & $\mathbf{5 0}$ & 16 & 56 & $\mathbf{7 8 . 0 0 \%}$ & $\mathbf{8 4 . 2 1 \%}$ \\
\hline $\begin{array}{l}\text { Nikon D810 DSLR } \\
\text { Camera (Body Only) }\end{array}$ & $\mathbf{9 0}$ & $\mathbf{5 3}$ & $\mathbf{7 8}$ & $\mathbf{9 0 . 0 0 \%}$ & $\mathbf{8 1 . 5 4 \%}$ \\
\hline $\begin{array}{l}\text { Nikon 1 AW1 14.2MP } \\
\text { Waterproof. }\end{array}$ & $\mathbf{5 0}$ & $\mathbf{1 0}$ & $\mathbf{1 3}$ & $\mathbf{8 9 . 0 0 \%}$ & $\mathbf{8 6 . 9 2 \%}$ \\
\hline $\begin{array}{l}\text { Bargains Depot USB } \\
\text { Cable Lead Cord }\end{array}$ & 45 & 19 & 39 & $93.00 \%$ & $\mathbf{8 . 9 5 \%}$ \\
\hline
\end{tabular}

We evaluated the system with two measures, precision and relevance, shown in Figure 4 Precision measures the number of relevant pages that were seen vs. the total number of pages that were seen. Relevance measures the number of relevant pages seen plus the number irrelevant pages not seen vs. the total number queried

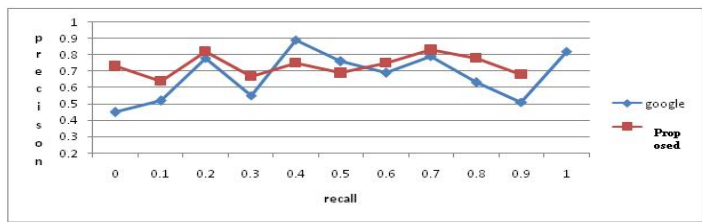

Figure 4 Precision Vs Recall graph for proposed system Vs GOOGLE

The next experiment aims at determining the importance of personalization by using generated dynamic user model during using the system. The user model is used to re-rank the retrieved documents to match the user interest

Personalization time:

Time to retrieve any information depends on the type of search engine, size of data set, relevancy between query and doc. User history \& re-ranking algorithm used.

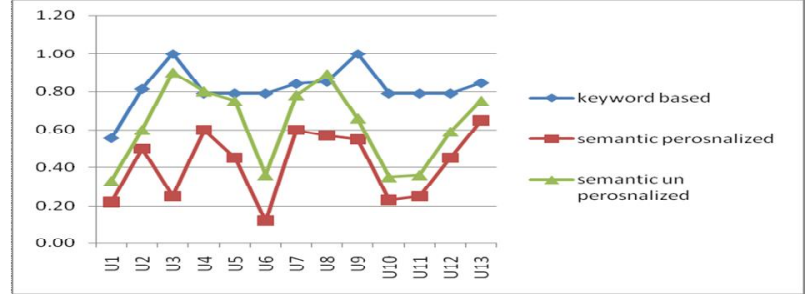

Figure 5 Performance efficiency of the new system

Figure 5 discuss the performance efficiency of the system when the system uses to retrieve the result.

It is observed that $80 \%$ users, out of 30 users in our data set, have found improved precision with the proposed approach in comparison to the standard search engine(Google) results, while $34 \%$ users have achieved equal precision with both approaches. It has been observed that users who posed Queries in unpopular context than well liked context got better performance. In addition, when the system can extract the exact context of user's need, the Precision and recall is found better than other search engine results.

\section{CONCLUSION AND FUTURE WORK}

In this paper, we propose a framework for semantic query manipulation and personalization of Electronic catalog service systems. We present the user profile ontology and its relation to other domain ontology's. Then, we explain the semantic query processing steps and present the result personalization steps. A complete scenario is illustrated to visualize the framework followed by experimental results. The empirical evaluation shows promising improvements in the relevancy of the retrieved results and of the user's satisfaction. It can be used in other domain by editing the domain ontology using export option of new system and building the domain concepts weight table .In future work, we will focus on: (1) automatically learn e-Catalog ontological concepts, properties and relationship from web to build PCO; (2) add business properties besides general properties to $\mathrm{SCO}$; (3) construct the Reasoning and Expending Module of ABPECSS, to set rules onto SCO.

\section{REFERENCES}

[1]. J. de Bruijn, D. Fensel, and M. Kerrigan, Modeling Semantic Web Services, Heidelberg: Springer-Verlag,2008, pp. 30-52.

[2] . E. Casasola, ProFusion personal assistant: An agent for personalized Information filtering on the WWW, M.S. thesis, The University of Kansas, Kansas, KCK, U.S.A., 1998.

[3]. I. Chen, J. Ho, and C. Yang, On hierarchical web catalog integration with conceptual relationships in

thesaurus, in Proceedings of the 29th Annual International ACM SIGIR Conference on Research and

Development in Information Retrieval, Washington, 2006, pp. 635-636.

[4]. O. Corcho, A. Gómez-Pérez, Solving integration problems of e-Commerce standards and initiatives through ontological mappings, in Proceedings of the 17th International Joint Conference on Artificial Intelligence,

Seattle, 2001.

[5]. R. Cyganiak, A relational algebra for SPARQL. Digital Media Systems Laboratory HP Laboratories Bristol. 
HPL-2005-170, September 28, 2005.

[6]. Z. Cui, D. Jones, and P. O'Brien, Semantic B2B Integration: Issues in Ontology-based Approaches, SIGMOD Record, vol. 31, no. 11, 2002.

[7]. S. Gauch, J. Chaffee, and A. Pretschner, Ontology-based personalized search and browsing, Web Intelligence and Agent Systems, vol. 1, no. 3-4, pp. 219-234, 2003.

[8]. L. Kwon and C. O. Kim, Recommendation of ecommerce sites by matching category-based buyer query and product e-Catalogs, Computers in Industry, vol. 59, no. 4, pp. 380-394, 2008.

[9] J. Lee and T. Lee, Massive catalog index based search for e-Catalog matching, in Proceedings of the 9thIEEE International Conference on e-Commerce Technology. Tokyo. IEEE Computer Society, 2007, pp. 341-348

[10]. H. Lee, J. Shim, S. Lee, and S. Lee, Modeling considerations for product ontology, in Lecture Notes in

Computer Science, Advances in Conceptual Modeling: Theory and Practice, vol. 4231, Tucson, AZ: Springer, 2006, pp. 291-300.

[11]. J. Leukel, V. Schmitz, and F. Dorloff, A modeling approach for product classification systems, in Proceedings of 13th International Conference on the Database and Expert Systems Applications. Aix-en-Provence, 2002, pp. 868-874.

[12]. H. Li, XML and industrial standards for electronic commerce, Knowledge and Information Systems, vol. 2,no. 4, pp. 487-497, 2000.

[13]. S. Liao, C. Chen, C. Hsieh, and S. Hsiao, Mining information users' knowledge for one-to-one marketing on information appliance, Expert Systems with Applications, vol. 36, no. 3, pp. 4967-4979, 2009.

[14]. L. Lim and M. Wang, Managing e-Commerce catalogs in a DBMS with native XML support, in Proceedings of the IEEE International Conference on e-Business Engineering, Beijing, 2005, pp. 564-571.

[15]. C. Lin and C. Hong, Using customer knowledge in designing electronic catalog, Expert Systems with
Applications, vol. 34, no. 1, pp. 119-127, 2008

[16]. D. Liu, Y. Lin, and C. Chen, Deployment of personalized e-Catalogues: An agent-based framework integrated with XML metadata and user models, Journal of Network and Computer Applications, vol. 24, no. 3, pp. 201228, 2001.

[17]. K. Masanobu, D. Kobayashi, D. Xiaoyong, and I. Naohiro, Evaluating word similarity in a semantic network,Informatics, 2000, vol. 24, no. 1, pp. 192-202.

[18]. H. Paik and B. Benatallah, Personalised organisation of dynamic e-Catalogs, in Web Services, e-Business, and the Semantic Web (C. Bussler, R. Hull, S. McIlraith, M. E. Orlowska, B. Pernici and J. Yang, Eds.).Heidelberg, Berlin: Springer Verlag, 2002, pp. 139-152.

[19] E. Prud'hommeaux and A. Seaborne. (2005, July) SPARQL Query Language for RDF. W3C Working Draft. [Online]. Available: http://www.w3.org/TR/2005/WD-rdfsparql-query-20050721/.

[20]. R. Rada, H. Mili, E. Bicknell, and M. Blettner, Development and application of a metric on semantic nets, IEEE Transaction on System, Man and Cybernetics, vol. 19, no. 1, pp. 17-30, 1989.

[21]. H. Sun-Young and K. Eun-Gyung, A study on the improvement of query processing performance of OWL data based on Jena, in Proceedings of the International Conference on Convergence and Hybrid Information Technology, Daejeon, 2008, pp. 678-681. [22]. A. Tversky, Feature of similarity, Psychological Review, vol. 84, no. 4, pp. 327-352, 1977.

[23] Dr.M.Thangaraj and Mrs. M.Chamundeeswari Agent Based personalized Semantic Web Information Retrieval System in (IJACSA) International Journal of Advanced Computer Science and Applications, Vol. 5, No. 8, 2014 